\title{
Offenbarung und Realität des Wissens bei Fichte
}

\author{
Kazimir Drilo
}

In der Zeit zwischen 1792 und 1806 entwickelt Fichte zwei unterschiedliche Bestimmungen des Offenbarungsbegriffs: 1. Offenbarung ist eine auf der göttlichen Autorität beruhende Lehre, mit der das sittliche Handeln gefördert werden soll; 2. Offenbarung ist das Zusammenhalten des Wissens im Sein, sie ist die schöpferische Tätigkeit, die das Wissen vor dem Absturz ins Nichts bewahrt und es im Sein hält. Offenbarung, so wie sie Fichte in seiner Spätphilosophie versteht, dient weder der Förderung der Moral noch wird sie als der Prozess der Selbstobjektivierung und der Selbsterkenntnis des Absoluten bestimmt. Der Begriff der Offenbarung hat vielmehr seine Bedeutung als der Garant der Realität des Wissens. Im Folgenden möchte ich skizzieren wie diese Neubestimmung des Offenbarungsbegriffs zu verstehen ist; als Beispiele dienen mir der Versuch einer Kritik aller Offenbarung, die Erlanger Wissenschaftslehre und die AzsL zum seligen Leben.

\section{Offenbarung als Förderung der Moralität}

In dem 1792 erschienen Versuch einer Kritik aller Offenbarung denkt Fichte den Begriff der Offenbarung im Hinblick auf seine Funktion für die Stärkung der Achtung vor dem Sittengesetz. Er unterscheidet zwei Fälle der Bestimmung des Willens durch den Willen Gottes, einmal durch die Naturreligion, zum zweiten durch die geoffenbarte Religion. Die Naturreligion gründet sich auf ein schon bestehendes Moralgefühl, so dass Gott dasjenige, das schon in uns liegt - die moralischen Anlagen - nur noch entwickeln muss; die geoffenbarte Religion begründet dagegen das Moralge- 
fühl, in ihr lässt Gott durch ein "geheimnisvolles übernatürliches Mittel « ${ }^{1}$ und durch seine Autorität etwas Neues und Unbekanntes in uns entstehen. ${ }^{2}$ Offenbarung ist eine »durch übernatürliche Kausalität von Gott in der Sinnenwelt hervorgebrachte Wirkung, durch welche sich Gott als der moralische Gesetzgeber ankündigt «. ${ }^{3}$ Sie wird als die Bestimmung des unteren Begehrungsvermögens durch das obere Begehrungsvermögen gedeutet, und zwar in denjenigen Fällen, in denen die Achtung vor dem Sittengesetz durch ein starkes Gegengewicht der Neigung eingeschränkt wird.

Als die Voraussetzung für das Ergehen der Offenbarung konstruiert Fichte den Fall der Existenz eines Volkes, bei dem die Kausalität des Sittengesetzes ganz oder zum großen Teil zum Erliegen gekommen ist. Dieses Volk besteht aus rohen und sinnlichen Menschen, bei denen "nicht einmal der Wille da ist, ein Moralgesetz anzuerkennen, und ihm zu gehorchen«, weil »die sinnlichen Triebe die einzigen Bestimmungsgründe ihres Begehrungsvermögens sind $« .{ }^{4}$ Diese sittliche Verrohung könne dann entstehen, wenn Völker durch Schicksalsschläge in die Lage kommen, im Kampf ums Überleben ihre Gedanken nur auf das, was »vor ihren Füßen lag, zu richten «, und so nur das Gesetz der Not kennenlernen. ${ }^{5}$ Bei den Menschen, die sich in tiefstem sittlichen Verfall befinden, entstehe nicht einmal der Wille, moralisch gut zu sein.

Doch wo soll die Offenbarung ansetzen, wenn nicht einmal der Wille da ist, moralisch zu handeln? Das Dilemma eines Offenbarungsbegriffs, der sich ausschließlich über seinen Nutzen für die Förderung der Moral definiert, formuliert Fichte folgendermaßen:

»Entweder fühlten die Menschen schon das sittliche Bedürfnis, das sie treiben konnte, eine Religion zu suchen, und hatten schon alle Moralbegriffe, die sie von den Wahrheiten derselben vernünftig überzeugen konnten; so bedurften sie keiner Offenbarung, sondern hatten schon a priori Religion: oder sie fühlten weder jenes Bedürfnis, noch hatten sie jene Be-

1 Fichte, Johann Gottlieb: Versuch einer Kritik aller Offenbarung. (Hg.) Hans Jürgen Verweyen, Hamburg ${ }^{\circ} 1983$, S. $^{\circ} 31$.

2 Fichte: Versuch, S. ${ }^{\circ} 35$

3 Fichte: Versuch, S. $^{\circ} 33$

4 Fichte: Versuch, $\mathrm{S} .{ }^{\circ}{ }^{\circ} 1$

5 Fichte: Versuch, ${ }^{\circ}{ }^{\circ} 51$ 
griffe; so konnten sie sich nie aus moralischen Gründen von der Göttlichkeit einer Religion überzeugen; aus theoretischen konnten sie es auch nicht; sie konnten es also überhaupt nicht, und eine Offenbarung ist folglich unmöglich." (Fichte, Versuch, S. 59)

Um aus diesem Dilemma herauszukommen nimmt Fichte an, dass letztendlich doch ein Bedürfnis nach Sittlichkeit schon vorhanden sein muss, damit eine Offenbarung überhaupt als solche erkannt werden kann. Dieses Bedürfnis sei jedoch den Menschen zunächst noch »wenig bewußt«; es reicht zwar nicht aus, um »zur Aufsuchung einer Religion getrieben « zu werden, aber es reicht dazu, um durch Hilfe der Offenbarung das Moralgefühl in sich zu entwickeln: "Es kündigte sich ihnen eine Lehre als göttlich an, und erregte dadurch wenigstens ihre Aufmerksamkeit." ${ }^{6}$ Aufmerksamkeit ist eine »empirische Bestimmung unserer Seele" und ist »durch natürliche Mittel zu erregen «. ' Obwohl von Fichte fast nur nebenbei erwähnt, spielt die Aufmerksamkeit eine wichtige Rolle: Sie ermöglicht dem noch unentwickelten Moralgefühl die Möglichkeit eine Offenbarung wahrzunehmen und sie anzunehmen, um so in den Prozess der Entwicklung des Moralgefühls einzutreten: »Ein vernünftiges Aufnehmen einer gegebenen Offenbarung, als göttlich, ist nur aus Gründen a priori möglich, aber a posteriori können, und müssen in gewissen Fällen, Gelegenheitsursachen gegeben werden, um diese Gründe zu entwickeln. ${ }^{8}$ Aufmerksamkeit ist, so wie die Einbildungskraft, diejenige »Kraft des Gemüts", die »von der einen Seite sinnlich, und also fähig ist, einer Bestimmung der sinnlichen Natur des Menschen entgegenzuwirken «, und von der anderen Seite »durch Freiheit bestimmbar ist, und Spontaneität hat «. ${ }^{9}$ Mit der »Aufmerksamkeit« findet Fichte einen Begriff, der auch in seiner Spätphilosophie von zentraler Bedeutung sein wird: Die Aufmerksamkeit auf das Leben des Absoluten ist die Bedingung für den angemessenen Nachvollzug der Wissenschaftslehre.

Mit seiner Theorie entfernt sich Fichte von Kant, in dessen Geist er die Offenbarungsschrift eigentlich verfassen wollte. Im Unterschied zu Fichte spricht Kant in seiner religionsphilosophischen Schrift - Die Religion in-

6 Fichte: Versuch, S. ${ }^{\circ} 60$

7 Fichte: Versuch, S. ${ }^{\circ} 58$

8 Fichte: Versuch, S. $^{\circ} 61$

9 Fichte: Versuch, S. ${ }^{\circ} 64$ 
nerhalb der Grenzen der bloßen Vernunft - dem Offenbarungsbegriff keine Bedeutung für die Moral zu. Eine Offenbarung, sei es als äußere oder als innere göttliche Mitteilung, in der die Naturgesetze oder die Autonomie der sittlichen Handlung aufgehoben werden, sei, so Kant, nur eine Art des Wunders, und somit kein Gegenstand der Philosophie. Zwar wird die Möglichkeit einer Offenbarung nicht bestritten, womit der Theologie ihr Recht eingeräumt wird, sie spielt jedoch weder für die sittliche Lebensführung noch für die Erkenntnis eine Rolle. Kant nennt die Offenbarung sogar eine Art von Wahnglauben, als den Glauben, »etwas durch Erfahrung zu erkennen, was wir doch selbst, als nach objektiven Erfahrungsgesetzen geschehend, unmöglich annehmen können «. ${ }^{10}$

Im Vergleich zu Kants klarer Absage an die Notwendigkeit einer Offenbarung bleibt ihr Stellenwert in Fichtes Frühschrift unklar. Der Versuch einer Kritik aller Offenbarung findet letztendlich doch keinen Ausweg aus dem angezeigten Dilemma, denn, um wirken zu können, muss die Offenbarung ein Bedürfnis nach Moralität voraussetzen - dann ist sie aber nur aus pädagogischen Gründen nützlich und letztendlich entbehrlich; außerdem muss sie auf das untere Begehrungsvermögen wirken, indem sie Aussicht auf die eigene Glückseligkeit beim Befolgen des Sittengesetzes eröffnet - in diesem Fall verunreinigt sie die Achtung vor der Heiligkeit des Gesetzes, die eigentlich allein willensbestimmend sein soll. Und wenn die Lehren der Offenbarung nur aufgrund von Gottes Autorität oder aus Angst vor Strafe befolgt werden, dann bekommt man nicht die Moralität, sondern nur die Legalität der Handlungen. Die Offenbarung gründet sich auf einem empirischen Bedürfnis. Obwohl sie außer uns ihre Realität hat, soll sie auf die Realität des Sittengesetzes in uns aufmerksam machen, ohne dieses in seiner Heiligkeit zu verdunkeln. Wie das Zugleich der Reinheit und der heiligkeit des Sittengesetzes und der auf die Sinne wirkenden Offenbarung zu denken ist, erklärt Fichte in seiner Frühschrift nicht. Aufmerksamkeit und Einbildungskraft bleiben in ihrer Vermittlungsfunktion zwischen Freiheit und Sinnlichkeit noch unbestimmt.

Erst mit der Neubestimmung des Wissensbegriffs kommt Fichte zu einem neuen und tieferen Verständnis der Offenbarung. Vorbereitet und be-

10 Kant, Immanuel: Die Religion innerhalb der Grenzen der bloßen Vernunft. (Hg) B. Stangneth, Hamburg ${ }^{\circ} 2003$, S. ${ }^{\circ} 263$ 
fördert wurde diese Entwicklung durch die nachkantischen Systementwürfe, die in den neunziger Jahren des 18. Jahrhunderts entwickelt wurden. Dieter Henrich hat in seinen Arbeiten, zuletzt in der Grundlegung aus dem Ich ${ }^{11}$, den Weg nachgezeichnet, auf dem die Tübinger Philosophen, an vorderster Stelle Schelling, durch die Verknüpfung von Jacobis Gedanken eines Unbedingten im Wissen, Spinozas Substanzbegriff und dem absoluten Ich aus Fichtes früher Wissenschaftslehre zu einer neuen Grundlage der Philosophie gelangt sind, die wiederum eine neue Theorie des Begriffs der Offenbarung zur Folge hatte. In diesem neuen Konzept ist die Offenbarung nicht mehr eine Lehre, die durch göttliche Autorität in einem bestimmten geschichtlichen Augenblick den Menschen zwecks Förderung der Moral mitgeteilt wird, sondern sie ist ein innergöttlicher, ewiger Prozess der Selbsterkenntnis und der Selbstobjektivierung des Absoluten:

"Der Prozeß als ganzer und dann auch eine jede Phase des Prozesses, in der das Bewusstsein seines Ursprungs im Unbedingten zum Durchbruch kommt, lässt sich als Offenbarung auffassen. Alle diese Offenbarungsereignisse unterscheiden sich von der Bedeutung von Offenbarung als Ergehen einer göttlichen Belehrung [ ]. Sie sind Weisen, in denen das Unbedingte als solches Eingang in das Wissen und Erfahren der Menschen findet.« (Henrich, Grundlegung, S. 866)

Auch Fichte verabschiedet den Offenbarungsbegriff seiner Frühschrift. In der Grundlage des Naturrechts von 1796 behält die Offenbarung jedoch noch ihre erzieherische Funktion. ${ }^{12}$ So heißt es, die Erziehung sei »Aufforderung zur freien Selbsttätigkeit" durch andere Selbstbewusstseine und habe eine fundamentale Funktion für die Bildung des eigenen Selbstbewusstseins. Sie sei konstitutiv für das Bewusstsein der Freiheit: »Alle Individuen müssen zu Menschen erzogen werden, außerdem würden sie nicht

11 Henrich, Dieter: Grundlegung aus dem Ich: Untersuchungen zur Vorgeschichte des Idealismus. Tübingen-Jena 1790-1794. Band I. Frankfurt a. M. ${ }^{\circ} 2004$, insb. S. ${ }^{\circ} 775-883$

12 Hansjürgen Verweyen sieht in den $\$ \$ 1-3$ der Grundlage des Naturechts schon einen Neuansatz Fichtes, da dort »als transzendentale Bedingung der Möglichkeit jedes individuellen Selbstbewusstseins [ ] der anerkennend-auffordernde Akt eines Vernunftwesens aufgewiesen [wird], in dem sich das angesprochene Ich erstmals seiner Freiheit bewusst werden kann.« Vgl. Verweyen, Hansjürgen: »Einleitung « zu: Johann Gottlieb Fichte, Versuch einer Kritik aller Offenbarung. (Hg.) Hans Jürgen Verweyen, Hamburg ${ }^{\circ} 1983$. S. $^{\circ}$ VII-LXXII ; hier S. ${ }^{\circ}$ XLI 
Menschen « ${ }^{13}$ Die erste erzieherische Tätigkeit geschah durch Got, der »das erste Menschenpaar " erzogen habe. ${ }^{14}$ Seitdem stehe jedes Individuum in der freien Wechselwirkung mit anderen Individuen. Die Realität der Welt sei die Bedingung des Selbstbewusstseins, "denn wir können uns selbst nicht setzen ohne etwas außer uns zu setzen, dem wir die gleiche Realität zuschreiben müssen, die wir uns selbst beilegen. ${ }^{15}$ Doch trotz dieser Aufwertung der intersubjektiv-erzieherischen Offenbarung, die man als Einübung in die Freiheit bezeichnen könnte, wird für Fichte in den folgenden Jahren die metaphysische, nur durch jeden einzelnen nachzuvollziehende Offenbarung wichtiger. Nur durch sie sind das Wissen vom Absoluten und das »selige Leben« möglich. In Fichtes Spätphilosophie geht es vor allem um das Verstehen dieser metaphysischen Offenbarung. Erst durch sie kommt Fichte zu einer Neubestimmung des Offenbarungsbegriffs, die sich sowohl von seinen frühen Ansätzen als auch von dem z. ${ }^{\circ}$ B. von Schelling entwickelten Offenbarungsbegriff im Sinne eines Prozesses der Selbstmanifestation des Absoluten unterscheidet.

\section{Fichtes Neubestimmung des Offenbarungsbegriffs}

Um das Jahr 1801 stellt Fichte seine Wissenschaftslehre auf neue Grundlagen. Das absolute Ich wird nicht mehr als das höchste Prinzip der Philosophie gedacht, sondern als von einer Realität abhängig, die nicht außerhalb oder jenseits des »Ich" ist, sondern die als und durch das »Ich « wirkt. Für Fichte ist diese Realität jedoch nicht ein innergöttlicher Prozess. Um seine Neubestimmung des Wissens- und Offenbarungsbegriffs besser zu verstehen hilft es sich anzuschauen, wie sie sich von Schellings Gedanken über das Absolute, so wie dieser sie in der Schrift Philosophie und Religion darstellt, unterscheidet. Bei der Beantwortung der Frage nach der Idee des Absoluten dürfe die Philosophie, so Schelling, nicht von dem Reflexionsstandpunkt ausgehen, um das Absolute zu erkennen; Fichte und Schelling haben sich in ihrem Briefwechsel gegenseitig vorgeworfen, der andere tue eben das, d. ${ }^{\circ}$. verbleibe auf dem Reflexionsstandpunkt und bekomme auf

13 Johann Gottlieb Fichte: GNR, Hamburg ${ }^{\circ}$ 1979; hier: S. ${ }^{\circ} 39$.

14 Ebd.

15 Fichte: Grundlage, S. ${ }^{\circ} 40$ 
diese Weise ein nur konstruiertes und kein wahres Absolutes. Von dem Standpunkt der Reflexion auszugehen bedeute, so Schelling, dass man »in der einen Hand das Ideale oder Subjective, in der anderen das Reale oder Objective hält, und beyde zusammenschlägt, so daß sie sich wechselseitig aufreiben, und das Product dieser Aufreibung dann das Absolute ist $«{ }^{16} \mathrm{Im}$ Absoluten gibt es jedoch keine Zusammensetzung oder Verknüpfung des Idealen und des Realen, des Subjektiven und des Objektiven. Das Absolute sei das schlechthin Ideale, und als solches und ohne weitere Vermittlung auch das schlechthin Reale. Indem es als ideal unmittelbar real ist, kann es nicht durch Erklärungen, sondern nur durch Anschauung erkannt werden. ${ }^{17}$ Die Anschauung ist der einzig angemessene Erkenntnisorgan der Seele, in ihr ist die Seele Eins mit Gott. Sie ist »eine bloß individuelle, aber in dieser Individualität doch eben so allgemeingültige Offenbarung, als es für den empirischen Sinn das Licht ist . $^{18}$

Die Schwierigkeiten dieses Konzeptes treten hervor, wenn Schelling zu erklären versucht, wie die endlichen Dinge aus dem Absoluten hervorgehen und wie sie sich $\mathrm{zu}$ ihm verhalten. In Schellings transzendentalen Theogonie ist das Absolute schlechthin ideal. Das schlechthin-Ideale ist gleich ewig mit der Form, die aber unter ihm steht. In diesem Modell ist das Reale eine Folge der ewigen Form, die wiederum eine Folge des Idealen ist. Der vielschichtige und subtile Gedankengang Schellings, der hier nicht wiedergegeben werden kann, mündet in der These, dass sich das Absolute in dem Realen kraft der Form selbst erkennt: "Das selbständige Sich-selbst-Erkennen des schlechthin Idealen ist eine ewige Umwandlung der reinen Idealität in Realität « ${ }^{19}$ Das Endliche entsteht durch die in der Selbsterkenntnis begründete Verdoppelung des Absoluten. Indem es sich selbst als Reales erkennt oder, wie es auch heißt, sich in das Reale hineinbildet, verleiht das Absolute dem Realen zwar die Freiheit und Selbständigkeit, eröffnet aber auch die Möglichkeit des Abfalls. ${ }^{20}$ Die Selbstobjekti-

16 Schelling, Friedrich Wilhelm Joseph: »Philosophie und Religion«, in: Ausgewählte Schriften in sechs Bänden. Band 3: 1804-1806. Frankfurt am Main ${ }^{\circ} 1985,{ }^{\circ}{ }^{\circ} 21-80 ;$ S. 32.

17 Schelling: Philosophie und Religion, S. ${ }^{\circ} 35 \mathrm{f}$.

18 Schelling: Philosophie und Religion, $\mathrm{S}^{\circ} 36$

19 Schelling: Philosophie und Religion, $\mathrm{S}^{\circ} 44$

20 Schelling: Philosophie und Religion, ${ }^{\circ}{ }^{\circ}$ of. 
vierung des Absoluten führt zur Entstehung eines Gegenbilds und zur Entfernung vom Urbild. In dieser Bewegung sind die Vorstellungen der Religion wie das Böse, der Sündenfall und die Versöhnung begründet. Die Selbstoffenbarung des Absoluten sei in dessen bewusstlosem Willen begründet, sich selbst zu gebären.

Für Fichte ist dieses Konzept einer Selbstoffenbarung des Absoluten, in dem das Absolute sich objektiviert, sich als Reales erkennt und von sich selbst abfällt, nichts anderes als Zeichen der Unfähigkeit »einen Akt ohne ein agirendes « $\mathrm{zu}$ denken, also der Unfähigkeit zum transzendentalen Denken: »Daß Er [Schelling, Vf.] einen Akt nicht anders zu denken vermag [außer mit einem agierenden Subjekt, Vf.], mag wohl seyn: es geht ausser ihm noch vielen andern eben so; dies ist die natürliche Blödsinnigkeit, schlechter Kopf, u. Mangel an der aller ersten Erforderniß zum Philosophiren ${ }^{21}{ }^{21}$ Schelling gelinge es nicht zu erklären wie die Realität in die schlechthinnige Idealität des Absoluten hineinkommt. Auf dem Weg, den Schelling eingeschlagen hat, ist diese Aufgabe nach Fichtes Ansicht auch gar nicht zu lösen, denn der Versuch, die Reflexionsform auf ein Absolutes hin zu übersteigen, das von ihr unabhängig ist, ist zum Scheitern verurteilt. Schellings Konzept der Selbstoffenbarung des Absoluten scheitere an der Unhintergehbarkeit der Reflexion. Nicht das Absolute, so Fichte, sondern unser Wissen muss der Gegenstand der philosophischen Untersuchung sein. Offenbarung des Absoluten sei nur als und durch das Wissen möglich, die Rede von der Selbstoffenbarung des Absoluten ist schon die Überschreitung des Wissens und somit nicht mehr der Gegenstand der Philosophie.

In zahlreichen Entwürfen der Wissenschaftslehre gelangt Fichte zu einer neuen Definition des Wissens. Sie lautet: Das Wissen ist die absolute Genesis, die immanente Kraft, das Bild des Absoluten, dessen äußeres Dasein oder Existenz. Auch das Absolute wird neu gedacht. Es besitzt keine Selbsterkenntnis. Es ist sich selbst »durchaus unzugänglich, rein in sich geschlossen und verborgen ${ }^{22}$ In einem Brief an Schelling schreibt Fichte: "Das absolute selbst aber ist kein Seyn, noch ist es ein Wissen, noch ist es Identität, oder Indifferenz beider: sondern es ist eben - das absolute - und

21 Fichte, Johann Gottlieb: Wissenschaftslehre 1805. (Hg.) Hans Gliwitzky, Hamburg ${ }^{\circ} 1984$, S. ${ }^{\circ} 48$. (= WL 1805).

22 Fichte, Johann Gottlieb: WL 1804-II, Hamburg ${ }^{\circ} 1975$, S. $^{\circ} 234$. (=). 
jedes zweite Wort ist von Uebel «. ${ }^{23}$ Aus dieser Bestimmung des Absoluten geht deutlich hervor, dass auch die Offenbarung nicht mehr als eine Offenbarung des Absoluten gedacht werden kann, denn das würde das Heraustreten des Absoluten aus sich und somit eine Differenz im Absoluten bedeuten, und das ist, so Fichte, unmöglich. Das Absolute kann nicht aus sich heraustreten oder sich irgendwie offenbaren oder äußern. Trotzdem bekommt der Offenbarungsbegriff bei Fichte eine spezifische Bedeutung im Zusammenhang mit der Analyse des Wissens. Im folgenden möchte ich in vier Schritten zusammengefasst wiedergeben, wie Fichte in der Erlanger Wissenschaftslehre von 1805 das Wissen und den Offenbarungsbegriff denkt. Diese Schrift ist wichtig, weil sie in der Formlehre den Zusammenhang von Wissen und Offenbarung herausarbeitet und einen deutlichen Bezug hat zu der etwas später entstandenen AzsL zum seligen Leben.

Die vier Schritte, in denen die Erlanger Wissenschaftslehre die Analyse des Wissens entwickelt, lauten: (1) Die Reflexionsform vernichtet die Realität des Wissens und führt zum Nihilismus; (2) Die Realität des Wissens erhält sich durch die Einsicht in die Grunddifferenz von Vollzugsaktualität und Reflexionsform, die wiederum als die metaphysische Offenbarung des Absoluten bezeichnet werden kann; (3) Jeder Philosophierende muss diese Einsicht selbst vollziehen, sie kann nicht andemonstriert werden; er muss auf sie aufmerksam werden; (4) Die Aufmerksamkeit auf das Leben des Absoluten in uns wird durch die historische Offenbarung in Gang gesetzt. Die Analyse des Wissens führt somit zu zwei Bestimmungen der Offenbarung, die sich gegenseitig durchdringen: Es sind die metaphysische Offenbarung, deren Genesis in der Wissenschaftslehre dargestellt ist und die historische Offenbarung, die durch das Christentum gestiftet wurde.

1. Zum ersten Schritt der Erlanger Wissenschaftslehre: Als erstes wird der dem Wissen innewohnende Nihilismus gezeigt. Der Nihilismus entsteht durch die Reflexionsform, die jeden Inhalt auflöst, so dass im Wissen nichts Reales mehr übrigbleibt. Hier nimmt Fichte Jacobis Kritik auf: Idealismus sei, so Jacobi, Wissen des Nichts, Nihilismus. Fichte stimmt dem zu. Er radikalisiert sogar den Vorwurf des Nihilismus, indem er die Welt und alle Dinge als das Nichts bestimmt. Diese Radikalität Fichtes muss man

23 Fichte an Schelling, 15. Januar 1802, in: Walter Jaeschke (Hg.): Transzendentalphilosophie und Spekulation. Der Streit um die Gestalt einer Ersten Philosophie (1799 1807), Quellenband. Hamburg ${ }^{\circ} 1993,{ }^{\circ} .{ }^{\circ} 153$ 
wörtlich nehmen: Alles was es gibt ist Nichts; nur das Absolute ist; das Absolute kann aber nicht aus ihm selber heraus gehen, denn jedes Herausgehen bringt es in eine Relation, die es vernichtet. Selbstverstehen, Selbstobjektivierung des Absoluten sind nicht möglich: »Wird nun aber diese Objektivität, durch das sich selbst verstehen, in die Form des Als aufgenommen, so geht die Realität wieder verloren, und das ganze Nichts tritt abermals ein ${ }^{24}$ Auf der Stufe der Reflexion bleibt die Welt vom Absoluten auf ewig getrennt. In dieser Trennung »liegt die unaustilgbare Spur ihres Nichts $« .{ }^{25}$ Fichtes Fazit lautet: "Leere Form, ohne allen Gehalt, reines Nichts überall, wohin wir bliken. Absoluter Idealismus «. ${ }^{26}$ Ohne ein Absolutes, das dem Wissen Realität gibt, gibt es nur die in sich selbst kreisende Tätigkeit der Reflexion, aus sich selber Inhalte zu erschaffen, um sie dann ins Nichts aufzulösen. Man könnte sagen: Im Gegensatz zu seiner frühen Wissenschaftslehre versteht Fichte »das Ich" nicht mehr als das sich schlechthin selber Setzende, sondern als das sich selber Vernichtende. Endlichkeit und Tod sind Grundmomente unseres Wissens.

Was ist aber das Mittel, dem Nihilismus des Wissens zu entgehen? Das Heilmittel besteht darin, bis zum Ende zu reflektieren. ${ }^{27}$ Wie das zu verstehen ist erläutert Fichte am Beispiel des Verhältnisses von Reflexion, die er als ein Grundmerkmal des Wissens die "absolute Reflektierbarkeit" nennt zu dem Glauben an das von der Reflexion unabhängige Absolute: Der Glaube an das Absolute »ist Unglaube an die absolute Reflektierbarkeit: er ist daher bedingt dadurch daß man diese, als absolut faktisch, erkenne, und als solche sie gelten lasse ${ }^{28}{ }^{28}$ Der Ausdruck $»$ daß man die absolute Reflektierbarkeit als absolut faktisch erkenne und als solche sie gelten lasse" ist Kritik an Schellings Vorgehen, das Fichte das »Kunststück « nennt, »zu einem vermeintlichen Absoluten zu kommen«, indem man willkürlich »irgendwo « zu reflektieren aufhört und dann an dieser Stelle den »Urgrund « des Wissens setzt. Diesem, wie Fichte es nennt »blinden Nichtreflektieren", das willkürlich mit der Reflexion aussetzt um Raum für das Absolute zu

\footnotetext{
24 Fichte: $W L$ 1805, S. ${ }^{\circ} 120$

25 Fichte: WL 1805, S. $^{\circ} 128$

26 Fichte: $W L$ 1805, S. ${ }^{\circ} 133$

27 Fichte: $W L$ 1805, S. ${ }^{\circ} 66$

28 Ebd.
} 
schaffen, ist das "Setzen u. stehen lassen der absoluten Reflektierbarkeit" entgegen zu halten, ohne "dem Scheine, den sie sich giebt«, zu glauben. ${ }^{29}$

2. Dem Stehenlassen der absoluten Reflektierbarkeit ohne jedoch an den allmächtigen Schein den sie sich gibt zu glauben geht die Einsicht auf, dass die Realität des Wissens auch im Nihilismus der Reflexion erhalten bleibt. Zu dieser Einsicht gelangt man, wenn man sich in den Mittelpunkt des Wissens stellt. Sich in den Mittelpunkt, in das Licht des Wissens zu stellen heißt zu der Einsicht in die Grunddifferenz des Wissens zu kommen: Das ist die Grunddifferenz von Vollzugsaktualität und Reflexionsform..$^{30}$ Die Realität des Wissens ist in dieser Grunddifferenz begründet. Das Absolute ist im Wissen anwesend als die in jeder Begriffsbestimmung, jeder Reflexionsform tätige Vollzugsaktualität (als Akt ohne agierendes Subjekt). Diese von der Reflexionsform unterschiedene Tätigkeit, das immerwährende Werden des Wissens und das Sich-Halten im Sein ist die Offenbarung des Absoluten. Es wird somit ein Absolutes im Wissen gedacht, von dem das Wissen zwar abhängig ist, das aber nicht `jenseits des Wissens ist, sondern das Licht, in dem sich das Wissen als reales erfährt. Fichte geht nicht von einer Theorie des Absoluten aus, um dann von dort aus das Moment der Differenz zu entwickeln, sondern er geht von einer Theorie des Wissens aus; im Unterschied zu Schelling will er sich innerhalb der Grenzen des Wissens halten, auf dem Standpunkt der Differenz.. Auf den Begriff der Offenbarung übertragen heißt das: Das Wissen ist immer schon offenbartes, im Licht des Absoluten stehendes. Das Faktum einer Offenbarung außer uns wird von Fichte in die Faktizität der Vollzugsaktualität des Wissens, also in die Offenbarung in uns umgewandelt. Offenbarung ist die Aktualität des Sich Formierens des Absoluten: »Das göttliche Existieren ist das absolute formieren seiner Existenz; oder das formieren seines innern Wesens zum Existieren«; gegen Schelling gerichtet

29 Fichte: $W L$ 1805, S. ${ }^{\circ} 67$

30 $\mathrm{Zu}$ der Differenz von Vollzugsmoment und Bestimmungsmoment vgl. Danz, Christian: "Im Anfang war das Wort: Zur Interpretation des Johannesprologes bei Schelling und Fichte«, in: Fichte-Studien, Bd. 8 (»Religionsphilosophie«). (Hg.) K. Hammacher - R. Schottky - W.H. Schrader, Amsterdam-Atlanta ${ }^{\circ}$ 1995. S. ${ }^{\circ} 21-39$; hier S. ${ }^{\circ} 33$ 
heißt es weiter: »Weltschöpfung: ganz richtig: nur nicht als factum factum, sondern als factum fiens $« .^{31}$

3. Die Struktur des Wissens muss anhand der transzendentalphilosophisch-genetischen Methode untersucht werden. Nur dasjenige, das vom individuell vollzogenen, lebendigen Akt der Vernunft aus als wahr erkannt wird, kann in der Wissenschaftslehre Bestand haben. Die transzendentalphilosophische Methode, so wie Fichte sie versteht, fordert, dass die Erkenntnisse der Untersuchung nicht nur gesagt, sondern auch energisch vollzogen werden. Jeder muss im Selbstvollzug des Wissens die Einsicht in die basale Differenz von Vollzugsaktualität und Reflexionsform selbst erzeugen und sie dann festhalten. Dass Gott - das absolute Sein - »in uns" existiert können wir nur dann erfahren, wenn wir die Bewegung der Wissenschaftslehre selbst vollziehen. Wenn wir sie in der ganzen Strenge, die ihrer Argumentation eigen ist vollziehen und dabei auf uns selbst achten, werden wir, so Fichte, zusammen mit der Selbstvernichtung der Reflexion das Aufscheinen des wahren Lebens, das Licht des Absoluten sehen. Nicht indem wir diese Einsicht behaupten und als ein Resultat der Untersuchung »objektiv hinstellen " leuchtet uns in der Reflexionsform das Absolute auf, sondern, wie Fichte betont, nur indem »wir es [das Licht] selbst werden ${\text {. } .{ }^{2}}^{2}$

In der Wissenschaftslehre von 1804 wird dem Vorgang des Selber zum Leben des Absoluten Werdens große Aufmerksamkeit geschenkt. Fichte bestimmt dort (genauso wie in der Wissenschaftslehre von 1805) das Leben des Absoluten als unser Leben. Wir sind immer schon das "Eine ungeteilte Sein « oder das Absolute: »Wir leben, eben unmittelbar im Lebensakte selber; wir sind daher das Eine ungetheilte Sein selber, in sich, von sich, durch sich, das schlechthin nicht herausgehen kann zur Zweiheit ${ }^{33}$ Wie kommen wir zu dieser Einsicht, wie vermeiden wir die Objektivierung des Absoluten? Fichtes Lösung sieht folgendermaßen aus: Wir wissen, dass wir Objektivieren, wissen aber auch unmittelbar, »daß diese Objektivität eben so wenig, als irgend eine andere, Etwas bedeutet $« .^{34}$ Wir sind $» j e n e s$ Wir, im unmittelbaren Leben selber", nicht durch ein »Etwas« charakterisiert, son-

31 Fichte: $W L 1805$, S. ${ }^{\circ} 106$

32 Fichte: $W L$ 1805, S. ${ }^{\circ} 134 \mathrm{f}$.

33 Fichte: WL 1804-II, S. ${ }^{\circ} 152$

34 Ebd. 
dern »lediglich durch unmittelbares, actuelles Leben selber «. ${ }^{35}$ Wenn wir eingesehen haben, dass die Objektivierung des Absoluten zwar notwendig sei, dass sie aber auch "Nichts gelte«, besteht unser Vorgehen darin, sie ruhig sich einstellen zu lassen, auf sie nicht zu achten und sie »vom Resultate abzuziehen. ${ }^{36}$

Diese, wie Fichte einräumt, »überraschende Einsicht« erfordert höchste Aufmerksamkeit. So wird der Zuhörer ausdrücklich »zu einer sehr tiefen Besinnung und Abstraktion " aufgefordert. ${ }^{37}$ Unsere Aufmerksamkeit ist das Licht und das Leben des Absoluten. Nur die aufmerksame Strenge und Energie des Denkens versetzt uns in die Perspektive des Absoluten und hält uns in ihr. Wir sind, so Fichte, durch die Aufmerksamkeit auf die Grunddifferenz des Wissens selber zum Vollzug, zum Leben des Absoluten geworden. Eine Beschreibung dieses Vollzugs sei nicht möglich, da jede Beschreibung Objektivierung ist. Das Sich-Halten im Vollzug des Absoluten ist ein Merkmal des absoluten Wissens, und in die Perspektive des absoluten Wissens »haben wir uns hineinzuversetzen « - das sei der absolute Zweck des Daseins. ${ }^{38}$

Vor dem Hintergrund der Tatsache, dass jedes weitere Wort außer »das Absolute ist " "von Übel ist «, darf das Absolute nicht als außerhalb des Wissens seiend bestimmt werden. Jacobis an Fichte gerichtete Kritik, "Gott ist, und ist außer mir, ein lebendiges, für sich bestehendes Wesen, oder ICH bin Gott. Es giebt kein drittes «, ${ }^{39}$ begegnet Fichte mit dem Satz: »Jene [die so, wie Jacobi, Gott entweder >außer uns setzen oder mit dem Wissen identifizieren] haben nur einen formalen Gott [..], der eigentliche reale Gott ist in uns selber . $^{40}$ Auf die Frage, wie wir diesen "realen Gott « in uns selber erkennen, können wir folgende Antwort geben: Erkenne in der Reflexion, die du jetzt, in diesem Augenblick, vollziehst, diejenige Kraft, die diese im Sein erhält, und erkenne diese Kraft als die Offenbarung Gottes in dei-

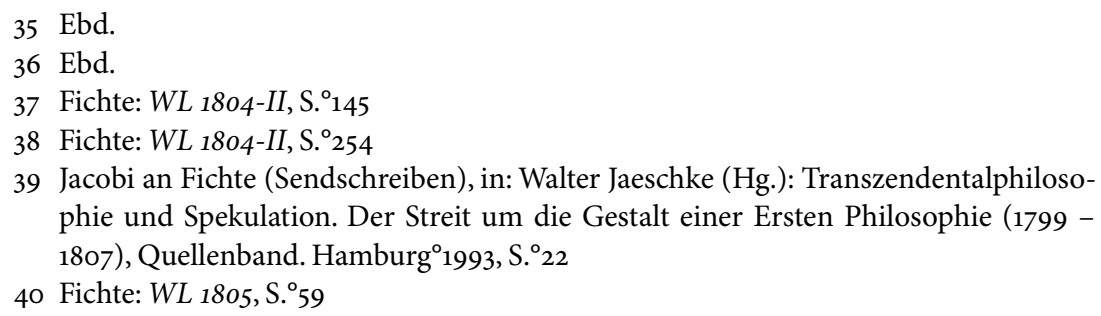


nem Wissen. Halte diese Erkenntnis fest und lasse nicht zu, dass sie vom Strom der Zeit ins Nichts fortgerissen wird. Werde selbst das Leben des Absoluten, werde selbst die Offenbarung des Absoluten. Wir sehen, dass in diesem Konzept die Aufmerksamkeit eine wichtige Rolle spielt, jedoch nicht mehr wie in der frühen Religionsschrift im Hinblick auf das in uns vorhandene Sittengesetz, sondern im Hinblick auf das in uns tätige Leben des Absoluten.

4. Dieser dritte Schritt verweist viertens auf die sittliche Kultivierung der Aufmerksamkeit. Die sittliche Kultivierung ist durch das Christentum, durch das historische Faktum der Offenbarung gegeben. Auf die Frage "wo ist denn nun das eigentlich Reale, das göttliche Existieren hingekommen « antwortet Fichte: "Darin ruht es, daß, und wie gelebt wird «. ${ }^{41}$ Dass gelebt wird und wie gelebt wird - in dieser doppelten Bedeutung besitzt der Offenbarungsbegriff der späten Wissenschaftslehre seinen Stellenwert: "Nur das absolute existiert [...]. Es existiert schlechthin, wie es muß, und weil es muß [..]. Wir aber sind ursprünglich sein unmittelbares Existieren selber, und können es auch in jedem Augenblicke der Zeit wirklich werden ${ }^{42}{ }^{42}$ Mit anderen Worten: Wir Menschen sind Offenbarungen Gottes, denn nur er existiert. Wir sind das jedoch nicht, indem wir bestimmte Eigenschaften besitzen oder bestimmte Lehren befolgen, sondern indem wir in jedem Augenblick die ins Nichts zerfließende Welt in Sein und im Bestehen zusammenhalten, also Gottes schöpferische Tätigkeit nachvollziehen.

In diesen vier Schritten - Aufweis des Nihilismus der Reflexionsform, Einsicht in die Grunddifferenz von Vollzugsaktualität und Reflexionsform, Aufmerksamkeit des Philosophierenden auf diese Einsicht, die Beförderung dieser Aufmerksamkeit durch das Christentum - vollendet sich Fichtes neuer Wissens- und Offenbarungsbegriff. 


\section{Historische und metaphysische Offenbarung}

Schauen wir uns noch an, wie Fichte in seiner wichtigsten religionsphilosophischen Schrift, der AzsL zum seligen Leben, dieses neue Konzept des Wissens darstellt. Die $A z s L$ ist nicht in erster Linie der Versuch, die Erkenntnisse der Wissenschaftslehre mit Hilfe eines populärphilosophischen Themas einem breiten Publikum mitzuteilen, sondern die Erläuterung der Frage nach der Bedeutung der Religion für die Kultivierung der Aufmerksamkeit und für das Verstehen der Wissenschaftslehre. Religion besitzt eine Schlüsselfunktion für die Wissenschaftslehre, denn sie bereitet den Boden vor, auf dem diese entstehen kann. Der Inhalt der wahren Religion und der Inhalt der Wissenschaftslehre unterscheiden sich nicht, der Unterschied bestehe nur in der Form, in der dieser Inhalt gewusst wird. Als Beispiel für den mit der Wissenschaftslehre identischen Inhalt der Religion führt Fichte den Johannesprolog an. In ihm sei der Inhalt die Wissenschaftslehre im Kern wiedergegeben. Der entscheidende Punkt ist für Fichte dabei die Unterscheidung von Gott und Wort, und zwar dergestalt, dass nur das Wort der Schöpfer der Welt ist und nicht Gott, dass es aber deshalb der Schöpfer ist, weil in ihm das göttliche Leben und das göttliche Licht ist, weil es - so können wir hinzufügen - Gottes Offenbarung als immerwährende Schöpfung ist. Zum Schöpfer der Welt wird das Wort jedoch nicht durch einen ursprünglichen, vor aller Zeit erfolgten »Akt absoluter Willkür «, sondern Gott und das Wort sind gleichursprünglich. ${ }^{43}$ In die Sprache der Wissenschaftslehre übertragen heißt das: Im Begriff (im Wort) ist die Vollzugsaktualität (das Absolute) tätig, sie übt durch den Begriff und als Begriff ihre schöpferische Tätigkeit aus.

Während Fichtes früher Offenbarungsbegriff nicht spezifisch christlich ist, sondern für alle drei monotheistische Religionen gilt, bekommt er in der Spätphilosophie eine eigentümliche christliche Fassung. Die Gedanken der Menschwerdung des Logos und der Einheit des göttlichen und des menschlichen Geistes werden zu Inbegriffen des neuen Wissens- und Offenbarungsbegriffs, der Johannesprolog wird zum Bezugspunkt nicht nur der Religionsphilosophie, sondern auch der Wissenschaftslehre. Allerdings

43 Fichte: Johann Gottlieb: Die AzsL zum seligen Leben. (Hg.) Hansjürgen Verweyen, Hamburg ${ }^{\circ}$ 2001, S. ${ }^{\circ} 91$ 
muss man festhalten, dass die zentralen Begriffe des Christentums - Gott als Person und Schöpfer, Trinität, Gedanken der Schuld, der Gnade und der Erlösung - in Fichtes Philosophie keine Rolle spielen. Für Fichte ist zwar das Wort der Schöpfer der Welt, es ist jedoch nicht mit dem Absoluten wesensgleich.

Im Zusammenhang mit der in der AzsL gestellten Frage nach dem seligen Leben ist es wichtig festzuhalten, dass von den fünf Grundprinzipien des Wissens, die Fichte entwickelt - das sind Sinnlichkeit, Moralität als Gesetzestreue, höhere Sittlichkeit, Religion und Wissenschaftslehre - nur die Religion die Sphäre des seligen Lebens ist. Sie ist »der innere Geist, der alles [... Denken und Handeln, durchdringt, belebt und in sich eintaucht ${ }^{44}{ }^{44}$ auch die Moralität und die Wissenschaftslehre. Im seligen Leben vereinigen sich das philosophische Wissen vom Absoluten und das religiös kultivierte Selbstgefühl. Seligkeit ist die Einheit des göttlichen und des menschlichen Geistes und die Kraft, durch die das Wissen vor Selbstvernichtung bewahrt wird: „Sobald er [der Mensch] sich aber rein, ganz, und bis in die Wurzel, vernichtet, bleibet allein Gott übrig, und ist alles in allem. Der Mensch kann sich keinen Gott erzeugen; aber sich selbst, als die eigentliche Negation, kann er vernichten, und sodann versinket er in Gott. «45

Als Beispiel für das selige Leben nimmt Fichte Jesus. Jesus war »die zu einem unmittelbaren Selbstbewußtsein gewordene, absolute Vernunft «, ${ }^{46}$ er schöpfte seine Erkenntnis »weder durch eigne Spekulation, noch durch Mitteilung von außen", sondern "hatte sie eben schlechthin durch sein bloßes Dasein «, »rein durch Inspiration ${ }^{47}{ }^{47}$ Insofern ist Jesus eine Ausnahme in der Geschichte, denn er lebte in der unmittelbaren Einheit mit Gott und gelangte zu dieser Einheit »lediglich historisch $"{ }^{48}$ nicht spekulativmetaphysisch. Wie das möglich sein soll, wo Fichte ausdrücklich betont, »nur das Metaphysische « - also die Wissenschaftslehre - »keineswegs aber das Historische, macht selig ${ }^{4}{ }^{49}$ bleibt indes unerörtert.

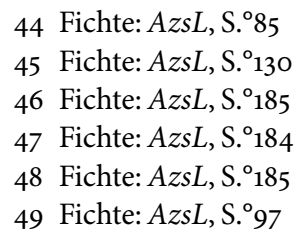


Die Charakterisierung, die hier von der Person Jesu Christi gegeben wird - er schöpfte seine Erkenntnis nicht durch die Spekulation, sondern durch sein bloßes Dasein - verweist auf ein ungelöstes Problem in Fichtes Religionsphilosophie. Fichte entwickelt keine Theorie, in der er das Verhältnis der historischen (erzieherisch-intersubjektiven) und der metaphysischen (sich in der Vollzugsaktualität des Wissens haltenden) Offenbarung klar bestimmt. Wie wichtig ist der Anstoß von außen für »das Metaphysische«, durch das der Mensch allein selig werden kann? Welchen Stellenwert hat die intersubjektiv-erzieherische Offenbarung für die von jedem einzelnen selbst zu vollziehende Wissenschaftslehre? Fichte kann der erzieherisch-intersubjektiven historischen Offenbarung auch keinen plausiblen Ort in seiner Geschichtsphilosophie zuweisen. So konstruiert er, zum Beispiel in den Grundzügen des gegenwärtigen Zeitalters, zum Teil abstruse Theorien von dem Aufkommen eines sogenannten "Normalvolks" oder »Urvolks" mit dem Sitz im »mittleren Asien «, an das die »Uroffenbarung « ergangen ist, und welches dann diese Lehre, durch die Vermischung mit einem anderen »Urvolk«, das zwar ohne die Offenbarung, jedoch mit einem starken Freiheitsbewusstsein ausgestattet ist, über Europa ausbreitet. ${ }^{50}$ Diese und weitere Schwierigkeiten entstehen dann, wenn Fichte die transzendentalphilosophische Methode verlässt, um die Geschichte kulturhistorisch zu deuten. Fichtes Philosophie verliert an Kraft, wenn sie »das Metaphysische " verlässt und sich »dem Historischen « zuwendet. Die transzendentale Methode der Wissenschaftslehre, die Religion und die Geschichte sind von Fichte in ihrem gegenseitigen Bezug nicht durchsichtig gemacht.

Trotz dieser offenen Fragen bleiben Fichtes Einsichten in das Wesen der Offenbarung immer noch bedenkenswert: Die Aufgabe der Philosophie besteht darin, die Struktur des Wissens zu analysieren; in der Analyse des Wissens leuchtet das Absolute auf - nicht als ein Gegenstand, eine Projektion, eine Idee, ein Postulat oder als der Urgrund des Wissens, sondern als die Kraft, die dem Trieb zur Selbstvernichtung des Wissens entgegenwirkt und dem Wissen Sein und Beständigkeit verleiht. Die Aufmerksamkeit und die Einsicht in diese schöpferische Kraft, ihr energischer Nachvollzug, ist die Offenbarung des Absoluten. Alle Versuche, Offenba- 
rung als Erziehung zur Moral, als Aufruf zur Freiheit oder als Prozess der Selbstmanifestation des Absoluten zu deuten bleiben mangelhaft, denn entweder suchen sie das Absolute außerhalb des Wissens statt in dem lebendigen Vollzug des Wissens selbst, oder sie klären das Verhältnis des Wissens zu dem Absoluten nicht, weil sie statt auf den Akt auf das agierende Subjekt (das Absolute) schauen und somit die Perspektive des Wissens verlassen.

Man kann sich fragen, ob das denn alles ist, was über die Offenbarung gesagt werden kann: sie sei die Vollzugsaktualität des Wissens? Darauf kann geantwortet werden, dass es für die Offenbarung wohl keine höhere Bedeutung geben kann als diejenige Kraft zu sein, durch die das Wissen vor dem Absturz in den Nihilismus bewahrt wird, dass es also nichts Wichtigeres und Grundlegenderes gibt als dem Wissen, das den Trieb hat, sich ins Nichts aufzulösen, Realität zu verleihen. 